\title{
Theoretical Studies of Hydrogen Storage Alloys
}

\author{
Final Report
}

Principal Investigator: Hannes Jonsson

Institution: University of Washington

Grant No.: DE-FG02-03ER46075

Budget Period: 09/15/2003 - 09/14/2006

Program Manager: Tim Fitzsimmons, Division of Materials Sciences and Engineering

\section{Summary}

Theoretical calculations were carried out to search for lightweight alloys that can be used to reversibly store hydrogen in mobile applications, such as automobiles. Our primary focus was on magnesium based alloys. While $\mathrm{MgH}_{2}$ is in many respects a promising hydrogen storage material, there are two serious problems which need to be solved in order to make it useful: (i) the binding energy of the hydrogen atoms in the hydride is too large, causing the release temperature to be too high, and (ii) the diffusion of hydrogen through the hydride is so slow that loading of hydrogen into the metal takes much too long. In the first year of the project, we found that the addition of ca. $15 \%$ of aluminum decreases the binding energy to the hydrogen to the target value of $0.25 \mathrm{eV}$ which corresponds to release of 1 bar hydrogen gas at 100 degrees C. Also, the addition of ca. $15 \%$ of transition metal atoms, such as $\mathrm{Ti}$ or $\mathrm{V}$, reduces the formation energy of interstitial H-atoms making the diffusion of $\mathrm{H}$-atoms through the hydride more than ten orders of magnitude faster at room temperature. In the second year of the project, several calculations of alloys of magnesium with various other transition metals were carried out and systematic trends in stability, hydrogen binding energy and diffusivity established. Some calculations of ternary alloys and their hydrides were also carried out, for example of $\mathrm{Mg}_{6} \mathrm{AlTiH}_{16}$. It was found that the binding energy reduction due to the addition of aluminum and increased diffusivity due to the addition of a transition metal are both effective at the same time. This material would in principle work well for hydrogen storage but it is, unfortunately, unstable with respect to phase separation. A search was made for a ternary alloy of this type where both the alloy and the corresponding hydride are stable. Promising results were obtained by including $\mathrm{Zn}$ in the alloy. 


\section{Results}

The primary goal of the project was to gain an understanding of the way in which metal alloys absorb and release hydrogen for the purpose of hydrogen storage in mobile applications and to search for promising candidate hydrogen storage materials. Below is a brief description of the key findings.

\section{Calculations of the binding energy and diffusion of hydrogen in pure magnesium metal and magnesium hydride}

We calculated the binding and diffusion of hydrogen in magnesium metal and magnesium hydride. Density functional theory was used to calculate the energy and atomic forces. The nudged elastic band and dimer methods were used to find rate and mechanism of hydrogen diffusion. The calculations agree well with available experimental data. The tetrahedral site is predicted to be more stable than the octahedral site for a hydrogen atom in the magnesium metal, but the optimal diffusion path goes through both sites. The diffusion is predicted to have activation energy of $0.22 \mathrm{eV}$ and should be fast at room temperature. In the hydride, $\mathrm{MgH}_{2}$, the diffusion is, however, extremely slow. The vacancy and interstitial mediated diffusion paths have activation energy of $2.3 \mathrm{eV}$ and 2.6 $\mathrm{eV}$, respectively, with the formation energy of the defect being the dominant contribution, $1.7 \mathrm{eV}$ and $2.3 \mathrm{eV}$, respectively. The energy per hydrogen atom in the hydride is, furthermore, $0.4 \mathrm{eV}$ lower than in the $\mathrm{Mg}$ metal. Together, these results help explain why the hydride tends to form a diffusion block near the surface if hydrogen is loaded quickly into the metal. The calculated charge density was analyzed with the Bader partitioning scheme which assigns a charge of -1.1 to -1.2 e on an interstitial hydrogen atom in the metal, but a smaller charge of -0.8 e on a hydrogen atom in the hydride.

\section{The effect of light impurities on the binding energy of hydrogen in magnesium metal and magnesium hydride}

With the goal of finding a way to reduce the temperature at which hydrogen gets desorbed upon heating, we have used density functional theory calculations to study how the binding energy and charge distribution of hydrogen atoms in magnesium and magnesium hydride are modified by the addition of impurities of some of the lighter elements, namely aluminium, silicium, sodium, carbon and boron. The binding energy is reduced by the addition of aluminium and silicium, elements that are more electronegative than magnesium, but increased by the addition of sodium, a less electronegative element. The most electronegative additives, carbon and boron, did not stay in substitutional sites in the magnesium lattice but spontaneously hopped into interstitial sites where they bind hydrogen atoms by strong covalent bonds. The charge of the interstitial hydrogen atoms as judged by the charge density partitioning scheme of Bader is -1.1 to -1.3 e in the magnesium metal, but -0.8 e in the hydride and is not affected strongly by the substitution of magnesium by the other elements. The most promising result was obtained by introducing aluminium into the magnesium lattice, as the binding energy of the hydrogen interstitial in the hydride was found to decrease linearly with the amount of aluminium and reach $0.2 \mathrm{eV}$ on average at about $15 \% \mathrm{Al}$. This is the target binding energy for hydrogen atom release at $300 \mathrm{~K}$. The weight percent of hydrogen in such an alloy would still be high, nearly the 
same as in pure $\mathrm{MgH}_{2}$. Such a material could possibly be made as an amorphous $\mathrm{Mg}_{x} \mathrm{Al}_{y}$ alloy, stabilized by a small amount of a third component.

\section{Theoretical studies of ternary magnesium-titanium hydride and magnesium-vanadium hydride}

The structure and hydrogen content of ternary magnesium-titanium hydride and magnesium-vanadium hydride crystals was determined by theoretical DFT calculations. The lattice constant and position of metal atoms agree well with the experimental X-ray data. The titanium based alloy is determined to have formula $\mathrm{Mg}_{7} \mathrm{TiH}_{1} 6$ with average hydrogen binding energy of $0.25 \mathrm{eV}$, consistent with the low desorption temperature measured experimentally. The hydrogen is predicted to be released at a 190 degree lower temperature than from pure magnesium hydride. This is in reasonably good agreement with the measured 150 degree shift in desorption temperature during rapid heating. The vanadium alloy is especially interesting in that vacancies are present in the metal atom lattice and additional hydrogen atoms are incorporated in octahedral holes adjacent to the vanadium atoms. The average hydrogen atom binding energy in the magnesium-vanadium hydride was determined to be $0.27 \mathrm{eV}$, again consistent with low desorption temperature observed in experiments.

4. The addition of transition metals dramatically increases the rate of hydrogen diffusion in magnesium based hydrides

The interstitial and vacancy mediatied diffusion of hydrogen in magnesium based hydrides was studied using density functional theory calculations and the nudged elastic band method to find minimum energy paths. The diffusion through pure magnesium hydride is very slow, both in the rutile and FCC type structures because the formation energy of the defects is high, over $2 \mathrm{eV}$. However, the substitution of one out of eight magnesium atoms for a $\mathrm{Ti}$ or $\mathrm{V}$ atom reduces the formation energy of interstitials by an order of magnitude and vacancies down to a quarter. The migration energy becomes somewhat larger, but the total activation energy is still much smaller when transition metal atoms are present and the diffusion rate is increased by more than ten orders of magnitude. Even for defects in the second and third site from the transition metal atom is the formation energy reduced. Analysis of the electronic density and orbitals shows that electron transfer takes places, even over large distances, between the transition metal ion and the interstitial.

\section{Trends in binding energy, diffusion rate and stability of $\mathrm{Mg}_{7} X \mathrm{H}_{16}$ hydrides}

We carried out DFT calculations of alloys and corresponding hydrides of formula $\mathrm{Mg}_{7} \mathrm{X}$ and $\mathrm{Mg}_{7} \mathrm{XH}_{16}$, resp., where $\mathrm{X}$ is one of the transition metals from the first row of transition metals in the periodic table. The goal was to study trends in hydrogen binding energy (which determines the release temperature), diffusion rate in the hydride (which determines the time needed to load the metal alloy with hydrogen) and stability of both the alloys and the hydrides. A wealth of information has been obtained which could be used to guide the search for new compounds. One particularly interesting lead is the use of $\mathrm{Zn}$ in such compounds. The hydrogen binding energy is particularly low, the diffusion 
still reasonably fast and the alloy and hydride are relatively more stable than for most of the other compounds.

\section{Personnel partially supported by the grant:}

1. Andri Arnaldsson - Ph.D. student

2. William Stier - post-doc (mostly funded by a fellowship from Petrolium Research Fund)

3. Hannes Jnsson - PI, part of summer salary.

\section{Invited presentation of the results at conferences:}

[1] National Meeting of the Am. Chem. Soc., Symposium on Modeling in Fuel Chemistry, San Diego, 2005.

[2] National Meeting of the Am. Chem. Soc., Symposium on Hydrogen Storage, San Francisco, 2006.

[3] International Symposium on Metal-Hydrogen Systems, Maui, Hawaii, 2006.

[4] Gordon conference on 'Hydrogen in metals', New Hampshire, USA, 2007.

\section{Publications describing the results:}

[1] W. Stier, L. G. Camargo, F. Óskarsson and H. Jónsson, 'Hydrogen storage in magnesium based alloys', Prepr. Pap.-Am. Chem. Soc., Div. Fuel Chem., vol. 50(1), page $15(2005)$.

[2] F. Óskarsson, E. S. Aradóttir and H. Jónsson, Calculations of release temperature of hydrogen storage materials, Prep. Pap. - Am. Chem. Soc. Div. Fuel Chem. 51, (2006). 